Randomized Trial

\title{
Effect of Individually Tailored Biopsychosocial Workplace Interventions on Chronic Musculoskeletal Pain and Stress Among Laboratory Technicians: Randomized Controlled Trial
}

Kenneth Jay, $\mathrm{MSc}^{1-3}$, Mikkel Brandt MSc ${ }^{1,4}$, Klaus Hansen, $\mathrm{BSc}^{1}$, Emil Sundstrup, MSc ${ }^{1,2}$, Markus Due Jakobsen, MSc ${ }^{1,2}$, mc schraefel, PhD ${ }^{3,5,6}$, Gisela Sjøgaard, Dr. Med. Sci., PhD², and Lars L. Andersen, PhD ${ }^{1,4}$

From: ${ }^{1}$ National Research Centre for the Working Environment, Copenhagen, Denmark; ${ }^{2}$ Department of Sports Science and Clinical Biomechanics, University of Southern Denmark, Odense, Denmark; ${ }^{3}$ Electronics and Computer Science, University of Southampton , United Kingdom; ${ }^{4}$ Physical Activity and Human Performance group, SMI, Department of Health Science and Technology, Aalborg University, DK-9220, Aalborg, Denmark 5 Roya Academy of Engineering Research Chair, London, United Kingdom; ${ }^{6}$ Engineering and Physical Sciences Research Council, Swindon, United Kingdom, EPSRC ReFresh Project, $\mathrm{EP} / \mathrm{Ko21907/1;}$

Address Correspondence: Kenneth Jay

National Research Centre for the Working Environment Lersø Parkallé 105 Copenhagen, Denmark

E-mail: kennethjay@icloud.com

Disclaimer: There was no external funding in the preparation of this manuscript.

Conflict of interest: Each author certifies that he or she, or a member of his or her immediate family, has no commercial association (i.e. consultancies, stock ownership, equity interest, patent/licensing arrangements, etc.) that might pose

a conflict of interest in connection with the submitted manuscript.

Manuscript received: 01-20-2015 Revised manuscript received: 05-19-2015 Accepted for publication: 05-28-2015

Free full manuscript: www.painphysicianjournal.com
Background: Chronic musculoskeletal pain is prevalent among laboratory technicians and work-related stress may aggravate the problem.

Objectives: This study investigated the effect of a multifaceted worksite intervention on pain and stress among laboratory technicians with chronic musculoskeletal pain using individually tailored physical and cognitive elements.

Study Design: This trial uses a single-blind randomized controlled design with allocation concealment in a 2-armed parallel group format among laboratory technicians. The trial "Implementation of physical exercise at the Workplace (IRMA09) - Laboratory technicians" was registered at ClinicalTrials.gov prior to participant enrolment.

Setting: The study was conducted at the head division of a large private pharmaceutical company's research and development department in Denmark. The study duration was March 2014 (baseline) to July 2014 (follow-up).

Methods: Participants ( $n=112$ ) were allocated to receive either physical, cognitive, and mindfulness group-based training (PCMT group) or a reference group (REF) for 10 weeks at the worksite. PCMT consisted of 4 major elements: 1) resistance training individually tailored to the pain affected area, 2) motor control training, 3) mindfulness, and 4) cognitive and behavioral therapy/education. Participants of the REF group were encouraged to follow ongoing company health initiatives. The predefined primary outcome measure was pain intensity (VAS scale 0 10) in average of the regions: neck, shoulder, lower and upper back, elbow, and hand at 10 week follow-up. The secondary outcome measure was stress assessed by Cohen's perceived stress questionnaire. In addition, an explorative dose-response analysis was performed on the adherence to PCMT with pain and stress, respectively, as outcome measures.

Results: A significant $(P<0.0001)$ treatment by time interaction in pain intensity was observed with a between-group difference at follow-up of $-1.0(95 \% \mathrm{Cl}:-1.4$ to -0.6$)$. No significant effect on stress was observed (treatment by time $P=0.16$ ). Exploratory analyses for each body region separately showed significant pain reductions of the neck, shoulders, upper back and lower back, as well as a tendency for hand pain

Within the PCMT group, general linear models adjusted for age, baseline pain, and stress levels showed significant associations for the change in pain with the number of physical-cognitive training sessions per week $(-0.60[95 \% \mathrm{Cl}-0.95$ to -0.25$])$ and the number of mindfulness sessions $(0.15$ [ $95 \% \mathrm{Cl} 0.02$ to 0.18$]$ ). No such associations were found with the change in stress as outcome.

Limitations: Limitations of behavioral interventions include the inability to blind participants to which intervention they receive. Self-reported outcomes are a limitation as they may be influenced by placebo effects and outcome expectations. 
Conclusions: We observed significant reductions in chronic musculoskeletal pain following a 10-week individually adjusted multifaceted intervention with physical training emphasizing dynamic joint mobility and mindfulness coupled with fear-avoidance and de-catastrophizing behavioral therapy compared to a reference group encouraged to follow on-going company health initiatives. A higher dose of physical-cognitive training appears to facilitate pain reduction, whereas a higher dose of mindfulness appears to increase pain. Hence, combining physical training with mindfulness may not be an optimal strategy for pain reduction.

Trial registration: NCT02047669

Key words: Mindfulness, dynamic joint mobility, work environment, fear avoidance, motor control exercise, strength, pain catastrophizing

Pain Physician 2015; 18:

U pper extremity musculoskeletal disorders account for $20-30 \%$ of all health ailments in the general working population (1-3). This is also problematic among laboratory technicians where typical daily work tasks include pipetting and preparing vial samples for analysis with subsequent computer processing. These activities result in extended periods of time spent in static postures, displaying sustained low force muscular contractions as well as repetitive precision movements requiring a high degree of motor control and concentration. Prevention or rehabilitation of neck and shoulder pain has been the focus of many workplace interventions, e.g., interventions with ergonomic changes (4) or physical exercise (5-8). Progressive resistance training - as a single element intervention strategy - has been successful and a clinically sound approach to counter work-related pain and tenderness from static and/or repetitive work (9-12). High-intensity resistance training, relying on principles of progressive overload, successfully reduced the incidence of non-chronic musculoskeletal pain of the upper extremity in occupational groups similar to laboratory technicians (13). However, when dealing with populations suffering from chronic (lasting more than 3 months) musculoskeletal pain in conjunction with psycho-physiological symptoms of stress-related pain and soreness, biopsychosocial interventions involving multifaceted comprehensive modalities may prove to be a more viable solution to reduce work- and stress-related musculoskeletal pain and discomfort.

Pain is a multifaceted combination of biological, psychological, and social factors integrated in a brain neuromatrix that feeds the outcome of pain perception in the individual (14-18). In a prospective cohort study among office workers without neck pain, stress approximately doubled the risk of future pain (19). Therefore, the pain and stress link should be taken into account as stress and work interactions are part of the psychological and social risk factors for pain development or chronicity. Designing combined multifactorial intervention treatments aiming at reducing chronic pain should take this into account as stress has been observed to influence emotional, physical, cognitive, and behavioral wellbeing. A biopsychosocial intervention strategy aimed at reducing musculoskeletal pain by acknowledging work stress as a potential factor in pain rehabilitation seems appropriate as psychosomatic research shows an isomorphic, consequence, and precursor stress-pain relationship (20). Therefore, one attractive multifactorial strategy to decrease the occurrence and intensity of musculoskeletal pain is to perform individual screenings and subsequently apply a combination of on-site physical and cognitive training personalized in such a way that the worker is offered targeted treatment in the area most needed, while simultaneously adjusting it to fit the individual's comfort level and limiting daily work task interference at the job site.

The aim of this study is to investigate the effect of a multifaceted worksite intervention - with individually tailored physical and cognitive elements - on pain intensity and stress level among laboratory technicians with chronic musculoskeletal pain.

\section{Methods}

\section{Study Design}

This trial uses a single-blind randomized controlled design with allocation concealment in a 2-armed parallel group format among laboratory technicians at a large private pharmaceutical company in Denmark. The participants were allocated to receive either physical/ mindfulness group-based training (PCMT group) or encouragements to follow on-going company health initiatives (REF group) for 10 weeks at the worksite. The study duration was March 2014 (baseline) to July 2014 (follow-up). 


\section{Ethics}

Ethical approval has been obtained from The Danish National Committee on Biomedical Research Ethics (the local ethical committee of Frederiksberg and Copenhagen; H-3-2010-062) as part of the research program "Implementation of physical exercise at the workplace (IRMA)." The trial "Implementation of physical exercise at the Workplace (IRMA09) - Laboratory technicians" was registered in the ClinicalTrials.gov registry prior to participant enrolment. Criteria of the revised Consolidated Standards of Reporting Trials (CONSORT) 2010 statement for reporting randomized trials have been followed (21). All experimental conditions conformed to The Declaration of Helsinki.

\section{Participant Recruitment and Study Flow}

A screening questionnaire on musculoskeletal pain and perceived level of stress was sent out to 752 laboratory technicians in a division of a large pharmaceutical company in Denmark. Inclusion criteria were being female and suffering from chronic musculoskeletal pain in one or more of the following regions: low back, upper back, neck, shoulder, elbow, or hand. In the present study we defined chronic pain as fulfilling all of the 3 following criteria in at least one of the 6 body regions: 1) the pain should have lasted at least 3 months, 2) pain intensity of $\geq 3$ ( $0-10$ Visual Analogue Scale [VAS]) during the last week, and 3) pain frequency of $\geq 3$ days during the last week (22). Life-threatening disease and pregnancy were considered contraindications to the physical testing and training and we therefore excluded such participants. All participants who met the inclusion criteria, were willing to participate in the trial, and did not meet exclusion criteria were subsequently invited for a clinical examination by a physical therapist ( $n=$ 184).

We did not exclude participants due to disease unless contraindications for all elements of the intervention existed. Instead, participants with typical exclusion criteria, e.g., hypertension, were allowed to participate in the less strenuous part of the intervention if their own doctor cleared them. All participants were informed about the purpose and content of the study and provided their written informed consent to participate.

After the clinical examination, randomization was performed among eligible participants $(n=112)$ who immediately thereafter received an email with their respective group allocation. Randomization was performed in SAS by allocating each participant a random number, sorting ascending, and merging with a con- secutive list of group PCMT, REF, PCMT, REF, etc. Fig. 1 shows the participant flow through the study. Table 1 shows baseline characteristics of the 2 groups after group allocation.

\section{Blinding}

All assessors (including statistical data processors) were kept blinded to which group the participants had been allocated. An ID number was given to the participants and coupled with their email initials, which was kept separate from the random numbers table providing the allocation. The participants were instructed not to reveal their particular intervention treatment at any point and only the physical trainer and mindfulness coach had knowledge of the participant allocation. The physical trainer and mindfulness coach were recruited as external consultants and were not at any point involved with other parts of the study. Blinding of the instructors and participants was not possible.

\section{Intervention}

The experimental intervention treatment (group PCMT) consisted of 4 major elements: 1) individualized motor control training, 2) individualized resistance training specific to the pain affected area 3) cognitive and behavioral modification education emphasizing individual specific concerns about pain and movement, and 4) general mindfulness. In short, the physical elements consisted of elastic resistance band exercises targeting the shoulder girdle and arm/hand, which previously has been shown to relieve musculoskeletal pain symptoms $(7,23,24)$, together with isolated and integrated slow and precise dynamic joint mobility exercises focusing on improving fine motor control skills (25-28). The psychological components of the intervention were guided mindfulness sessions, consisting of meditation and body scans, together with gentle yoga techniques and encouragement to practice at home. Physical training was coupled with cognitive and behavioral modification education focusing on pain de-catastrophizing (29-32) and fear-avoidance beliefs (33-37). Additionally, the psychological elements also involved pain management education and information also grouped in the physical training sessions. Based on the screening questionnaire replies, each participant was informed about her own stress score relative to average values and as to which body regions fulfilled the criteria for chronic pain. The physical training instructor also received the information about body regions with chronic pain for each individual. The purpose of this 


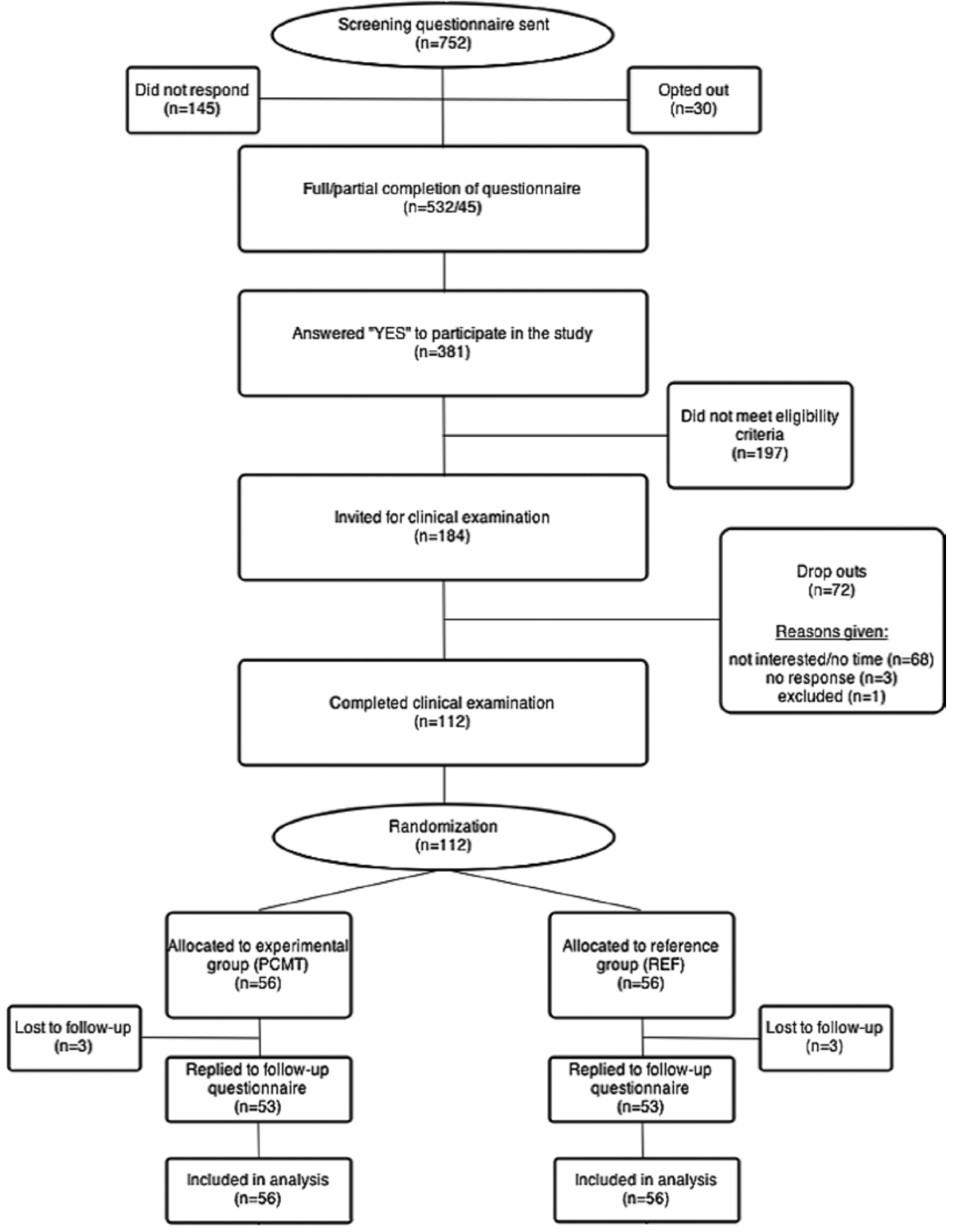

Fig. 1. Participant flow through the study. 
Table 1. Baseline characteristics of the 2 groups. ${ }^{*} P<0.05$. Participants in the 2 groups were not significantly different at baseline.

\begin{tabular}{|c|c|c|}
\hline & Group PCMT mean (SD) & Group REF mean (SD) \\
\hline \multicolumn{3}{|l|}{ Baseline characteristics } \\
\hline Age, years & $45.5(9.0)$ & $47.6(8.2)$ \\
\hline Height $(\mathrm{cm})$ & $163.4(7.2)$ & $164.1(6.8)$ \\
\hline Weight $(\mathrm{kg})$ & $65.5(14.3)$ & $65.8(11.4)$ \\
\hline Body Mass Index, kg.m-2 & $24.5(3.4)$ & $24.1(4.2)$ \\
\hline Smokers (percentage) & 11 & 9 \\
\hline Seniority (years) & $20.2(10.3)$ & $22.4(9.7)$ \\
\hline Working hours per week & $35.6(7.4)$ & $37.9(2.3)$ \\
\hline Average pain intensity of all regions & $2.9(1.5)$ & $2.6(1.4)$ \\
\hline \multicolumn{3}{|l|}{ Regional pain intensity } \\
\hline Neck & $3.5(2.7)$ & $3.5(2.6)$ \\
\hline Upper back & $2.7(2.8)$ & $2.4(2.7)$ \\
\hline Lower back & $3.7(2.6)$ & $3.1(3.1)$ \\
\hline Shoulder & $4.0(2.5)$ & $4.0(2.5)$ \\
\hline Elbow & $1.6(2.3)$ & $1.4(2.7)$ \\
\hline Hand & $1.9(2.5)$ & $1.3(2.3)$ \\
\hline \multicolumn{3}{|c|}{ Pain duration by region $>\mathbf{3}$ months (percentage) } \\
\hline Neck & 62.5 & 67.9 \\
\hline Upper back & 48.2 & 48.2 \\
\hline Lower back & 66.1 & 55.4 \\
\hline Shoulder & 62.5 & 67.9 \\
\hline Elbow & 28.6 & 17.9 \\
\hline Hand & 37.5 & 23.2 \\
\hline Stress score (0-40) & $15.5(7.0)$ & $13.9(5.9)$ \\
\hline
\end{tabular}

was to allow individual tailoring of the exercises and making the physical and cognitive programs more meaningful and relevant for the individual. The conceptual model for this intervention was that work may cause stress and musculoskeletal pain, but physical training and mindfulness can improve pain and stress, respectively (38), and thereby also have an indirect effect on stress and pain, respectively, as they are mutually influenced as recently suggested (39) (Fig. 2).

The REF group received a single email after randomization with encouragement to participate in the company's on-going health initiatives, e.g., weekly elastic band group training sessions (only available in some departments) and was encouraged to continue to take "active breaks" whenever needed. As this is part of the existing and currently on-going program at the company it can be considered "usual care." No new interventions were added in this group.

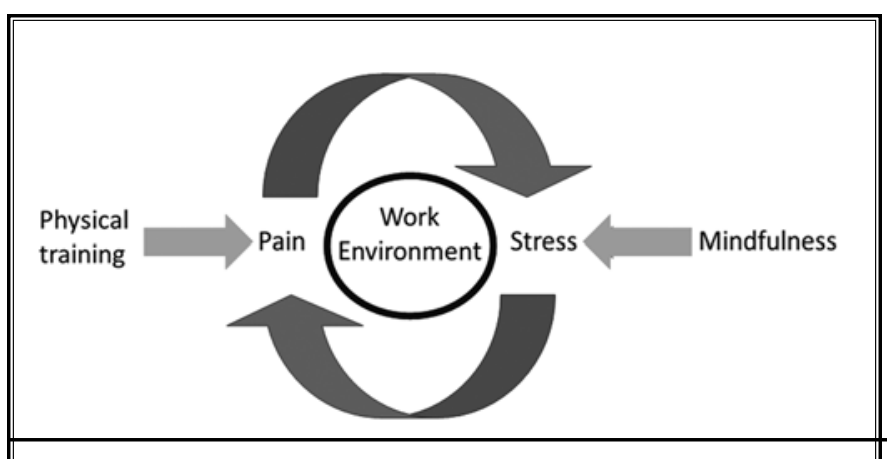

Fig .2. Conceptual model of the stress-pain relationship.

Jay et al (40) has previously described the intervention in detail.

Intervention Organization at the Work Site

Participants in the PCMT group could participate in supervised physical training sessions of 20 - minute duration on 
Monday, Tuesday, Thursday, and Friday. Three different time slots were available during each day to allow flexibility in relation to working schedules. Guided mindfulness sessions were made available once per week at 2 different times during the day each lasting 50 minutes. The schedule has previously been described elsewhere by Jay et al (40).

\section{Co-interventions}

Participants of both groups were recommended to continue their usual physical activities alongside the intervention. Furthermore, the company's own health and safety professionals were available to provide ergonomic education in accordance with the standard company policy, which consisted of ergonomic worksite observations by trained professionals and subsequent individualized recommendations on changing taskspecific positions and adjusting ergonomic aids to fit each department structure. Each individual department management was responsible for prioritizing and utilizing the option of ergonomic support in accordance with company policy.

\section{Primary Outcome}

A questionnaire with drawings of the upper extremity from the Nordic questionnaire (41), together with a horizontally oriented modified VAS scale (42) was used to identify changes between groups in preand post-intervention musculoskeletal pain and represents the primary outcome of this trial. For reference, a change in pain intensity of 1 on a scale of 0 to 10 is considered the minimal relevant difference in patients with chronic musculoskeletal pain, and a change of 2 is considered to be moderately clinically meaningful (43).

\section{Secondary Outcomes}

Our secondary outcome of perceived stress was measured by Cohen's perceived stress questionnaire (scale $0-40$ ) at baseline and follow-up. Further, an explorative dose-response analysis was performed on the adherence to physical training and mindfulness training with pain and stress, respectively, as outcome measures.

\section{Adverse Events}

During the intervention all instructors were asked to collect information regarding any adverse events of the training during each training session. Furthermore, the training instructors were available to provide individual email support and guidance to the participants if deemed necessary.

\section{Sample Size}

A priori power analysis based on previous measurements reveals that 27 participants of each group for $95 \%$ power, type I error probability of $5 \%$, SD of 1.5 and a minimal relevant difference in pain intensity of 1.5 is sufficient to test the null-hypothesis of equality (alpha $=0.05$, beta $=0.05$ ). Given an estimated $10 \%$ dropout rate, we aimed at recruiting at least 30 participants for each group.

\section{Statistical Analysis}

We performed all statistical analyses in accordance with the intention-to-treat principle by including all participants in the analysis regardless of actual participation or dropout. Missing values were not imputed but handled by the statistical software. Pain and stress between-group differences at follow-up were determined by a linear mixed model using Proc Mixed of the SAS statistical software, version 9.2 (SAS institute, Cary, NC, USA). Fixed factors were group, time, and group by time interaction. Analyses of pain and stress were controlled for pain and stress at baseline, respectively.

Within the PCMT group, general linear models were used to determine the dose-response association between the change in the outcome variables and the number of physical-cognitive training sessions per week (range $0-3$ ) and the total number of mindfulness sessions (range $0-8$ ). Analyses were adjusted for age and baseline pain and stress levels.

Results are reported as least square means (95\% Cl). We accepted $P$-values less than 0.05 as statistically significant. Baseline and descriptive results are reported as mean (SD) and between-group differences as least square means $(95 \% \mathrm{Cl})$.

\section{REsults}

PCMT adherence were 2.1 (0.9) times per week for physical training and 3.8 (2.4) times total for mindfulness during the intervention period, which corresponds to $70 \%$ and $47.5 \%$ of the intended sessions, respectively.

No adverse events were reported during the intervention. Three people of each group were lost to follow-up (Fig. 1). Two participants changed jobs and the remaining 4 participants dropped out with no reason given.

A significant $(P<0.0001)$ group by time interaction in pain intensity (average of all included regions) was observed with a between group difference at follow-up of $-1.0(95 \% \mathrm{Cl}:-1.4$ to -0.6$)$. No significant (group by time $P=0.16$ ) effect on stress was observed. 
Table 2. Changes in pain intensity (primary outcome; average of neck, upper and lower back, shoulder, elbow, and hand) and perceived stress (secondary outcome) from baseline to 10-week follow-up. Differences for each group are shown separately on the left, and contrasts between groups on the right. The P-value shown is for the group by time interaction. Values are least square means (95\% CI).

\begin{tabular}{|l|c|c|c|c|}
\hline & \multicolumn{4}{|c|}{ Difference from baseline to follow-up } \\
\hline Outcome measure & $\begin{array}{c}\text { Within group } \\
\text { (PCMT) }\end{array}$ & $\begin{array}{c}\text { Within group } \\
\text { (REF) }\end{array}$ & $\begin{array}{c}\text { Between group difference at } \\
\text { follow-up } \\
\text { (PCMT vs. REF) }\end{array}$ & P-value (group by time) \\
\hline Pain intensity (0-10) & $-1.5(-1.8$ to -1.1$)$ & $-0.3(-0.7$ to 0.0$)$ & $-1.0(-1.4$ to -0.6$)$ & $<0.0001$ \\
\hline Stress $(0-40)$ & $-2.5(-3.8$ to -1.2$)$ & $-1.2(-2.5$ to 0.2$)$ & $-1.1(-2.4$ to 0.3$)$ & 0.16 \\
\hline
\end{tabular}

Table 3. Results of explorative analysis on pain of the specified regions. Changes in pain intensity for each region of neck, upper and lower back, shoulder, elbow, and hand from baseline to 10-week follow-up are shown. Differences for each group are shown separately on the left, and contrasts between groups on the right. The P-value shown is for the group by time interaction. Values are least square means $(95 \%$ CI). ns = non-significant.

\begin{tabular}{|l|c|c|c|c||}
\hline & \multicolumn{4}{|c|}{ Difference from baseline to follow-up } \\
\hline $\begin{array}{c}\text { Regional pain } \\
\text { intensity }\end{array}$ & $\begin{array}{c}\text { Within group } \\
\text { (PCMT) }\end{array}$ & $\begin{array}{c}\text { Within group } \\
\text { (REF) }\end{array}$ & $\begin{array}{c}\text { Between group difference at } \\
\text { follow-up (PCMT vs. REF) }\end{array}$ & P-value (group by time) \\
\hline Neck & $-1.5(-2.2$ to -0.9$)$ & $-0.2(-0.8$ to 0.6$)$ & $-1.3(-2.0$ to -0.6$)$ & 0.0051 \\
\hline Upper back & $-1.5(-2.1$ to -0.9$)$ & $-0.3(-0.9$ to 0.3$)$ & $-1.1(-1.7$ to -0.5$)$ & 0.0072 \\
\hline Lower back & $-1.7(-2.4$ to -1.0$)$ & $-0.1(-0.7$ to 0.6$)$ & $-1.5(-2.2$ to -0.8$)$ & 0.0007 \\
\hline Shoulder & $-2.2(-2.9$ to -1.6$)$ & $-0.6(-1.2$ to 0.1$)$ & $-1.6(-2.3$ to -0.9$)$ & 0.0007 \\
\hline Elbow & $-0.9(-1.3$ to -0.4$)$ & $-0.6(-1.1$ to -0.2$)$ & $-0.1(-0.6$ to 0.3$)$ & $\mathrm{ns}$ \\
\hline Hand & $-1,0(-1.5$ to -0.4$)$ & $-0.3(-0.8$ to 0.3$)$ & $-0.5(-0.5$ to 0.1$)$ & $\mathrm{ns}$ \\
\hline
\end{tabular}

Within-group pain reductions of the PCMT and REF groups were were $52 \%[-1.5(95 \% \mathrm{Cl}:-1.8$ to -1.1$)$ and $15 \%$ [-0.3 (95\% Cl: -0.7 to 0.0$)$ ], respectively. For stress, the within-group changes were $17 \%[-2.5(95 \% \mathrm{Cl}:-3.8$ to -1.2$)]$ and $8 \%$ [-1.2 (-2.5 to 0.2$)]$ for PCMT and REF groups, respectively (Table 2 ).

Subsequently, an explorative analysis was performed on changes in pain for each of the 6 body regions. For neck, upper back, lower back, and shoulder pain significant between group changes in pain were observed of $-1.3(-2.0$ to -0.6$),-1.1(-1.7$ to -0.5$),-1.5(-2.2$ to -0.8$)$ and $-1.6(-2.3$ to -0.9$)$ (all 4 regions $P=0.05$ ), respectively (Table 3 ).

Within the PCMT group, general linear models adjusted for age, and baseline pain and stress levels showed significant associations for the change in pain with the number of physical-cognitive training sessions per week $(-0.60$ [95\% Cl: -0.95 to -0.25$])$ and the number of mindfulness sessions ( 0.15 [95\% Cl: 0.02 to 0.18$])$. That is, a higher number of physical training sessions were associated with reduced pain, whereas a higher number of mindfulness sessions were associated with increased pain. No such associations were found with the change in stress as outcome.

\section{Discussion}

This randomized controlled trial showed positive effects on self-reported musculoskeletal pain intensity following 10 weeks of combined physical training and mindfulness with cognitive behavioral therapy and pain management/education at the worksite. The intervention did not reduce stress significantly as measured by Cohen's perceived stress questionnaire. Finally, the dose-response analysis showed a contrasting effect of physical training and mindfulness on pain with physicalcognitive training, reducing pain 0.60 points for each physical training session per week (range $0-3$ ) and mindfulness sessions increasing pain 0.15 per mindfulness session (range $0-8$ ). Thus, the difference between performing 0 and 3 physical-cognitive training sessions per week corresponds to a pain reduction of 1.8 and the difference between 0 and 8 mindfulness sessions corresponds to a pain increase of 1.2. 


\section{Musculoskeletal Pain}

Our results showed a significant reduction in musculoskeletal pain compared with the reference group following the intervention. An essential part of interpreting these results concerns their clinical relevance. In that regard, congruency between studies exists when discussing clinically meaningful changes in pain (44). Changes of 1.7 points in pain intensity on a $0-10$ scale have been argued by Farrar and coworkers (45) as a valid discrimination between patients with pain who, on the patient global impression of change scale, improved much or very much and patients who did not improve or worsened. Comparably, Dworkin et al (43) reviewed that a change in pain intensity of 2 points is moderately clinically meaningful and a difference of one point is minimally clinically relevant. However, while these numbers refer to individual changes in pain, research studies commonly report average changes of several individuals. Thus, the results of our study can be considered clinically relevant especially considering that the pain score is an average of 6 areas whereas only one area was required to meet chronic pain criteria to be included. Our results on pain is congruent with results of several other studies investigating musculoskeletal pain and the effects of physical exercise $(23,24,46,47)$. For instance, Andersen et al (24) showed moderate reductions in pain, tenderness, and headache with as little as 2 minutes of daily resistance training performed at the workplace in office workers experiencing frequent neck and shoulder pain. Similarly Ahlgren et al (7) found that regular resistance training, endurance training, and co-ordination training can also alleviate musculoskeletal pain in populations suffering from trapezius myalgia. We have also shown positive effects and clinically relevant reductions in musculoskeletal pain in the low back and neck following resistance-training interventions. For instance, Jay et al (48) showed approximately $50 \%$ reductions in neck and low back pain following 8 weeks of group-based kettlebell training at the work site in a group of female laboratory technicians. Likewise, Sundstrup et al (49) showed a clinically relevant reduction of 1.5 points ( 0 - 10 scale) in shoulder, arm, and hand pain (average) compared to controls following 10 weeks of progressive resistance training in slaughterhouse workers.

What separates the current study from previous studies is the emphasis on precise dynamic joint mobility exercises targeting the joints of the affected areas. The strength training element only accounted for a small portion of each training by focusing on one set of 10 repetitions without going to muscular exhaustion of approximately $3-4$ exercises using elastic resistance bands as described elsewhere (40). Each training session in the current study lasted approximately 20 minutes, leaving ample time to perform dynamic joint mobility exercises focusing on precision of movement execution similar to tai chi performance (40). Considering the average pain rating of the investigated areas was relatively low at baseline, the observed reduction may suggest that other factors than muscular strengthening can assist in the modulation of pain. One suggestion as to the effects of precise dynamic joint mobility on musculoskeletal pain may be found in a change in the activity of nociceptive and mechano receptive afferents from the targeted joint and muscles in combination with higher order central nervous system modulation as described in Melzack and Wall's Gate Control Theory more than 40 years ago (50). One idea suggests that during precise, slow, and focused dynamic joint mobility training more mechanoreceptors are activated in the joint capsules causing the brain to modulate the output of pain, as more mechano receptive afferents are firing and thereby minimizing the influence of nociceptive input on pain interpretation in the brain. Tai chi has been shown to help alleviate acute low back pain in young men and a recent systemic review by Ye et al (51) showed positive effects of tai chi in pain patients with knee osteoarthritis. Further, Segura-Jiménez et al (52) found positive effects of tai chi on acute pain in patients suffering from fibromyalgia. The precise, focused, and slow dynamic joint mobility emphasized in the training intervention of the present study was inspired by tai chi movement and principles as previously described (40) and may therefore, at least partly, explain the significant pain reduction observed.

\section{Stress}

The lack of significant stress reduction in the present study is congruent with recent findings of van Berkel et al (53). The study by van Berkel (53) was published after commencement of our trialour trial, therefore we could not include their experience in our study design. The group looked at changes in lifestyle behaviors in 129 workers receiving mindfulness training, e-coaching, daily physical activity in the form of walking, and fruit, and compared it to a control group. At 6- and 12- month follow-up no changes in lifestyle behaviors could be detected, thus concluding that the effectiveness of a worksite mindfulness-based multi-component intervention could not be shown (53). While van Berkel et al (53) evaluated the effect of mindfulness on behav- 
ioral changes affecting the workers' lifestyle to become more active, they did not evaluate the effect on stress. In our study we used mindfulness with the aim of reducing stress, which also involved behavioral changes by becoming more self-aware of stressful situations, the effects it has on the body, and how to normalize the stress response. The effects of mindfulness-based stress reduction in relation to quality of life, mood, symptoms of stress, and immune parameters have previously been investigated in a group of cancer patients showing enhanced quality of life and decreased stress symptoms following a similar, but more intense, intervention protocol (54). Furthermore Carlson et al (54) showed positive adaptations in immune profile following the intervention. Although the population in the present study is different as we investigated the effects of mindfulness on generally healthy workers with pain symptoms, the positive effects of mindfulness-based interventions towards stress have been quite thoroughly investigated. Several recent systemic reviews (55-57) of randomized controlled trials investigating mindfulnessbased stress reduction and mindfulness-based cognitive behavioral therapy in populations with clinical and non-clinical psychosomatic diagnosis concluded that mindfulness-based stress reduction and behavioral therapy improve mental health and reduce the incidence of depressive relapse with medium effect sizes. However, the lack of rigorous designs of these studies makes this conclusion dubious. As Fjorback et al (57) notes the included review studies lack active control groups and long-term follow-ups. This may partly explain why we did not observe a significant stress reduction compared with the reference group in our study. Another systemic review on mindfulness-based stress reduction (58) found, in general, that mindfulness-based stress reduction and cognitive therapy have efficacy as adjunctive interventions for anxiety symptoms in patients with diagnosed conditions. Additionally, mindfulnessbased stress reduction was found beneficial for general psychological health and stress management in those with other medical and psychiatric illnesses as well as in healthy individuals. Finally, Marchand (58) concludes that mindfulness stress reduction and meditation have a role in pain management. With 2 systemic reviews on the efficacy of mindfulness-based stress reduction both concluding that it has beneficial effects on stress management and coping, it is somewhat interesting that we could not detect a significant change in stress in the present study. Possible explanations may include the difference in measuring methods, populations, in- tervention durations, as well as frequencies of guided mindfulness and it cannot be ruled out that a longer intervention period with mindfulness would have contributed positively to a reduction in perceived stress. However given the results of the dose-response in the present study, it is likely that mindfulness may have had better efficacy had the participants not been suffering from chronic pain. In our study with generally healthy individuals with musculoskeletal pain, the stress level at baseline was relatively low. Thus, a flooring effect may exist where changes in stress cannot be detected. As another possible explanation, performing mindfulness together with colleagues for almost an hour during the workday while the job tasks pile up may not provide the calm atmosphere necessary for such an intervention. Consequently, our study shows that an implementation at the worksite with one weekly guided mindfulness session lasting 50 minutes is inadequate to reduce the level of self-perceived stress over the course of 10 weeks in a group of female laboratory technicians. Considering the results of van Berkel et al (53) who provided 90 minutes of weekly guided mindfulness at the worksite, e-coaching, and $5 \times 30$ minutes of weekly practice at home over the course of 8 weeks and still could not show significant effects on lifestyle behavior, implementing mindfulness at the worksite may not be a viable solution to reduce stress at work, at least not in the current implemented format.

\section{Stress-Pain and Dose-Response Relationship}

As the biopsychosocial model shows interdependence of biological, psychological, and social factors, the idea of reducing stress as a method to reduce musculoskeletal pain is attractive. However, in the current study design, and in contrast to our expectations, we could not show a decrease in stress between groups. It is however interesting to speculate whether a successful reduction in stress would have additionally contributed to the reduction in pain. For example, if participants were getting stressed because of musculoskeletal pain (consequence stress-pain relationship), we would expect that by reducing pain, we could also reduce stress. This was, however, not the case. Conversely, if the participants were experiencing pain because of stress, we would expect to see a decrease in pain together with a decrease in stress. Since we did not observe a decrease in stress but still found a decrease in pain, we cannot conclude a precursor stress-pain relationship either. Regardless of the lack of significant results on stress in the present study, the stress-pain relationship remains 
an interesting theory that deserves to be explored in greater detail. This is supported by the current position on the multifaceted theory of pain as a complicated brain neuromatrix $(15,16,59)$.

Much to our surprise, the explorative dose-response analysis suggested that a combination treatment with group-based mindfulness sessions, in addition to not alleviating stress, impacted the physical and cognitive training negatively and thereby reduced the overall positive effect of the PCMT intervention.

The general idea of how mindfulness meditation exerts its effects is by neuroplasticity creating enhanced self-regulation. This process involves i) attention regulation, ii) body awareness, iii) emotion regulation (including reappraisal and exposure, extinction, and reconsolidation), and iv) change in perspective on the self (60). Neuroimaging has shown neuroplastic changes in the anterior cingulate cortex, insula, temporo-parietal junction, fronto-limbic network, and default mode network structures all involved in cognitive and behavioral modulation. For instance, Zeidan and colleagues (61) looked at possible brain mechanisms to support the modulation of pain with mindfulness meditation. They found that mindfulness meditation reduced painrelated activation of the contralateral primary somatosensory cortex. Furthermore, meditation-induced reductions in pain intensity ratings were associated with increased activity in the anterior cingulate cortex and anterior insula, areas involved in the cognitive regulation of nociceptive processing (61).

Kabat-Zinn et al (62) showed significant reductions in measures of present-moment pain, negative body image, inhibition of activity by pain, mood disturbance, and psychological symptomatology, including anxiety and depression, with a 10-week mindfulness meditation program in 90 chronic pain patients compared to a reference group receiving a traditional treatment protocol. If mindfulness has been shown to increase body awareness and overall body self-regulation and that is the process by which mindfulness could help alleviate pain, it is interesting that our dose-response analysis showed an opposite effect - that participation in mindfulness sessions slightly increased pain perception for each session attended. A possible explanation to our results is that the increased body awareness made the participants more attentive towards what they were feeling - including nociceptive input. The increased awareness towards musculoskeletal pain during a busy working day as a laboratory technician may not have been beneficial as participants still needed to use the pain affected areas of their bodies while having become more aware of the nociceptive signals following the mindfulness session. Further, our participants, while having chronic pain, were still working full time. The results may have been different if the participants had not had to work at all during the intervention period. Hence, the setting at the worksite and additional workrelated circumstances may influence the effect, or lack thereof, of mindfulness in pain reduction.

\section{Strengths and Limitations}

An important strength of the present study is the single-blinded randomized controlled design. In addition, the number of participants of each group was approximately twice as many as required to show relevant changes in pain. Cross contamination can diminish between group-differences in workplace trials, and can largely be avoided by randomizing the workplace at department level. However, this also decreases statistical power due to the inflation factor associated with clustering. In the present study only $10 \%$ of all employees participated (i.e., those with chronic pain and willing to participate), which minimizes the risk of cross contamination compared with all employees participating. Furthermore, instructors ensured that only participants randomized to training and mindfulness session participated in these sessions, thereby eliminating between-group contamination.

Limitations of behavioral interventions include the inability to blind participants to which intervention they receive. Self-reported outcomes are a limitation as they may be influenced by placebo effects and outcome expectations. Nevertheless, pain is a subjective experience, and the reference group was not a pure control group as they were encouraged to participate in the ongoing activities at the company and continue usual leisure time physical activities during the intervention, which allows for a range of variety in activity level. However, as the groups were not statistically different in key parameters at baseline, the leisure time activity can therefore be assumed to be similar during the intervention in the 2 groups. Finally, the intervention was comprised of several different elements, which makes it impossible to determine which part(s) of the intervention had the primary effect on the reduction in pain we observed. Therefore, we performed a post hoc dose-response analysis to assess the effect of the individual elements of intervention. 


\section{Conclusion}

We have shown clinically relevant reductions in chronic musculoskeletal pain following a 10-week individually adjusted multifactorial intervention with physical training emphasizing dynamic joint mobility and mindfulness coupled with fear-avoidance and de-catastrophizing behavioral therapy compared to a reference group following on-going company health initiatives.

These results suggest that work-related chronic musculoskeletal pain can be significantly reduced with physical training consisting of elastic band exercises and dynamic joint mobility coupled with cognitive behavioral education but combining it with mindfulness may not be an optimal strategy, as it appears to increase pain perception and counter the effects of the physical training.

\section{Acknowledgments}

Thanks to Thomas La Cour and Nina Sommer for supervising the physical/cognitive training and mindfulness, respectively.

A special thanks to Tina Nissen for valuable logistical help at the worksite.

We would like to thank the editorial board of Pain Physician and to the Royal Academy of Engineering, EPSRC ReFresh Project, EP/K021907/1; for review and constructive criticism in improving the manuscript.

\section{Author Contributions}

LLA had full access to all the data in the study and takes responsibility for the integrity of the data and the accuracy of the data analysis. KJ and LLA designed the study protocol, recruited the company, and obtained the funding. GS provided constructive feedback on the protocol. KJ, LLA, MB, ES, and MDJ designed the experimental test procedure. $\mathrm{KJ}, \mathrm{MB}$, and $\mathrm{KH}$ collected the data. KJ managed the literature searches and summaries of previous related work and wrote the manuscript with inputs from all other authors. LLA was responsible for statistical analysis. LLA, GS, MB, ESU, MDJ, KH, and $\mathrm{mc}$ provided revision for intellectual content and final approval of the manuscript.

\section{Conflict of Interest}

No authors have any conflicts of interest to report. None of the authors of the manuscript received any remuneration. Further, the authors have not received any reimbursement or honorarium in any other manner.

\section{Funding/Support}

The authors wish to disclose and thank the sponsor/ funding agent of the study. The study was funded by Novo Nordisk A/S Research and Development Department, Måløv, Denmark. The funding corresponds to a level 6 of the Pain Physician author guidelines. Novo Nordisk had no influence or interference after the protocol was designed.

\section{References}

1. Punnett L, Wegman DH. Work-related musculoskeletal disorders: The epidemiologic evidence and the debate. J Electromyogr Kinesiol 2004; 14:13-23.

2. Larsson B, Søgaard K, Rosendal L. Work related neck-shoulder pain: A review on magnitude, risk factors, biochemical characteristics, clinical picture and preventive interventions. Best Pract Res Clin Rheumatol 2007; 21:447-463.

3. Bot SDM, van der Waal JM, Terwee CB van der Windt DAWM, Schellevis FG, Bouter LM, Dekker J. Incidence and prevalence of complaints of the neck and upper extremity in general practice. Ann Rheum Dis 2005; 64:118-123.

4. Samani A, Holtermann A, Søgaard K, Madeleine P. Following ergonomics guidelines decreases physical and cardiovascular workload during cleaning tasks. Ergonomics 2012; 55:295-307.

5. Viljanen M, Malmivaara A, Uitti J, Rinne M, Palmroos P, Laippala P. Effectiveness of dynamic muscle training, relaxation training, or ordinary activity for chronic neck pain: Randomised controlled trial. BM] 2003; 327:475.

6. Hagberg M, Harms-Ringdahl K, Nisell R, Hjelm EW. Rehabilitation of neck-shoulder pain in women industrial workers: A randomized trial comparing isometric shoulder endurance training with isometric shoulder strength training. Arch Phys Med Rehabil 2000; 81:1051-1058.

7. Ahlgren C, Waling K, Kadi F, Djupsjöbacka M, Thornell LE, Sundelin G. Effects on physical performance and pain from three dynamic training programs for women with work-related trapezius myalgia. J Rehabil Med 2001; 33:162-169.
8. Ylinen J, Takala E-P, Nykänen $M$, Häkkinen A, Mälkiä E, Pohjolainen T, Karppi $\mathrm{SL}$, Kautiainen $\mathrm{H}$, Airaksinen O. Active neck muscle training in the treatment of chronic neck pain in women: A randomized controlled trial. JAMA 2003; 289:2509-2516

9. Andersen LL, Andersen $\mathrm{CH}$, Sundstrup E, Jakobsen MD, Mortensen OS, Zebis MK. Central adaptation of pain perception in response to rehabilitation of musculoskeletal pain: Randomized controlled trial. Pain Physician 2012; 15:385-394.

10. Andersen LL, Christensen KB, Holtermann A, Poulsen OM, Sjøgaard G, Pedersen MT, Hansen EA. Effect of physical exercise interventions on musculoskeletal pain in all body regions among office workers: A one-year randomized con- 
trolled trial. Man Ther 2010; 15:100-104.

11. Andersen LL, Jakobsen MD, Pedersen MT, Mortensen OS, Sjøgaard G, Zebis MK. Effect of specific resistance training on forearm pain and work disability in industrial technicians: Cluster randomised controlled trial. BMJ Open 2012; 2:e000412.

12. Coury HJCG, Moreira RFC. Evaluation of the effectiveness of workplace exercise in controlling neck, shoulder and low back pain: A systematic review. Rev Bras Fisioter 2009; 13:461-479.

13. Zebis MK, Andersen LL, Pedersen MT, Mortensen $\mathrm{P}$, Andersen $\mathrm{CH}$, Pedersen MM, Boysen M, Roessler KK, Hannerz $\mathrm{H}$, Mortensen OS, and Sjøgaard G. Implementation of neck/shoulder exercises for pain relief among industrial workers: A randomized controlled trial. BMC Musculoskelet Disord 2011; 12:205.

14. Moseley G. A pain neuromatrix approach to patients with chronic pain. Man Ther 2003; 8:130-140.

15. Khalsa PS. Biomechanics of musculoskeletal pain: Dynamics of the neuromatrix. J Electromyogr Kinesiol 2004; 14:109-120.

16. Melzack R. Pain and the neuromatrix in the brain. J Dent Educ 2001; 65:1378-1382.

17. Gatchel RJ, Peng YB, Peters ML, Fuchs PN, Turk DC. The biopsychosocial approach to chronic pain: Scientific advances and future directions. Psychol Bull 2007; 133:581-624.

18. Derbyshire SWG. Exploring the pain "Neuromatrix" Curr Rev Pain 2000; 4:467-477.

19. Hush JM, Michaleff Z, Maher CG, Refshauge $K$. Individual, physical and psychological risk factors for neck pain in Australian office workers: A 1-year longitudinal study. Eur Spine ] 2009; 18:1532-1540.

20. Arena JG, Sherman RA, Bruno GM, Smith JD. The relationship between situational stress and phantom limb pain: Cross-lagged correlational data from six month pain logs. J Psychosom Res 1990; 34:71-77.

21. Moher D, Hopewell S, Schulz KF, Montori V, Gøtzsche PC, Devereaux PJ, Elbourne D, Egger M, Altman DG . CONSORT 2010 Explanation and Elaboration: Updated guidelines for reporting parallel group randomised trials. J Clin Epidemiol 2010; 63:e1-e37.

22. Andersen LL, Mortensen OS, Hansen JV, Burr $\mathrm{H}$. A prospective cohort study on severe pain as a risk factor for long-term sickness absence in blue- and whitecollar workers. Occup Environ Med 2011; 68:590-592.

23. Andersen LL, KjÆr M, SØgaard K, Hansen L, Kryger Al, SjØgaard G. Effect of two contrasting types of physical exercise on chronic neck muscle pain. Arthritis Care Res (Hoboken) Wiley Online Library 2008; 59:84-91.

24. Andersen LL, Saervoll CA, Mortensen OS, Poulsen OM, Hannerz $\mathrm{H}$, Zebis MK. Effectiveness of small daily amounts of progressive resistance training for frequent neck/shoulder pain: Randomised controlled trial. Pain 2011; 152:440-446.

25. Schmidt R, Lee T. Motor Learning and Performance, 5 E With Web Study Guide: From Principles to Application. Human Kinetics, Champaign IL, USA 2013.

26. Wulf G. Gabriele Wulf on attentional focus and motor learning. Bewegung und Train 2007; 1:1-64.

27. Lohse KR, Sherwood DE, Healy AF. On the advantage of an external focus of attention: A benefit to learning or performance? Hum Mov Sci 2014; 33:120-134.

28. Wulf G, Shea C, Lewthwaite R. Motor skill learning and performance: a review of influential factors. Med Educ 2010; 44:75-84.

29. Swinkels-Meewisse IEJ, Roelofs J, Oostendorp R a B, Verbeek ALM, Vlaeyen JWS. Acute low back pain: Pain-related fear and pain catastrophizing influence physical performance and perceived disability. Pain 2006; 120:36-43.

30. McWilliams LA, Asmundson GJG. The relationship of adult attachment dimensions to pain-related fear, hypervigilance, and catastrophizing. Pain 2007; 127:27-34

31. Hutting N, Staal JB, Heerkens YF, Engels JA, Nijhuis-van der Sanden MWG. A self-management program for employees with complaints of the arm, neck, or shoulder (CANS): Study protocol for a randomized controlled trial. Trials 2013 14:258.

32. Holtermann A, Jørgensen MB, Gram $B$, Christensen JR, Faber A, Overgaard $\mathrm{K}$, Ektor-Andersen J, Mortensen OS, Sjøgaard G and Søgaard K. Worksite interventions for preventing physical deterioration among employees in job-groups with high physical work demands: Background, design and conceptual model of FINALE. BMC Public Health 2010; 10:120.

33. Leeuw M, Goossens MEJB, Linton SJ, Crombez G, Boersma K, Vlaeyen JWS.
The fear-avoidance model of musculoskeletal pain: Current state of scientific evidence. J Behav Med 2007; 30:77-94.

34. Vlaeyen JWS, Linton SJ. Fear-avoidance model of chronic musculoskeletal pain: 12 years on. Pain 2012; 153:1144-1147.

35. Lethem J, Slade PD, Troup JDG, Bentley $G$. Outline of a fear-avoidance model of exaggerated pain perception-I. Behav Res Ther 1983; 21:401-408.

36. Meulders A, Vansteenwegen D, Vlaeyen JWS. The acquisition of fear of movement-related pain and associative learning: A novel pain-relevant human fear conditioning paradigm. Pain 2011; 152:2460-2469.

37. Jørgensen $M B$, Ektor-Andersen J, Sjøgaard G, Holtermann A, Søgaard K. A randomised controlled trial among cleaners--effects on strength, balance and kinesiophobia. BMC Public Health 2011; 11:776.

38. Freitas-Swerts FCT de, Robazzi ML do CC. The effects of compensatory workplace exercises to reduce work-related stress and musculoskeletal pain. Rev Lat Am Enfermagem 2014; 22:629-636.

39. Bonzini M, Bertu' L, Veronesi G, Conti $M$, Coggon D, Ferrario MM. Is musculoskeletal pain a consequence or a cause of occupational stress? A longitudinal study. Int Arch Occup Environ Health 2014; 88:607-612.

40. Jay K, Brandt M, Sundstrup E, Schraefel MC, Jakobsen MD, Sjøgaard G, Andersen LL. Effect of individually tailored biopsychosocial workplace interventions on chronic musculoskeletal pain, stress and work ability among laboratory technicians: Randomized controlled trial protocol. BMC Musculoskelet Disord 2014; 15:444.

41. Kuorinka I, Jonsson B, Kilbom A, Vinterberg $\mathrm{H}$, Biering-Sørensen $\mathrm{F}$, Andersson G, Jørgensen K. Standardised Nordic questionnaires for the analysis of musculoskeletal symptoms. Appl Ergon 1987; 18:233-237.

42. Pincus T, Bergman M, Sokka T, Roth J, Swearingen C, Yazici Y. Visual analog scales in formats other than a 10 centimeter horizontal line to assess pain and other clinical data. J Rheumatol 2008; 35:1550-1558.

43. Dworkin RH, Turk DC, McDermott MP, Peirce-Sandner S, Burke LB, Cowan P, Farrar JT, Hertz S, Raja SN, Rappaport BA, Rauschkolb C, Sampaio C. Interpreting the clinical importance of group differences in chronic pain clinical tri- 
als: IMMPACT recommendations. Pain 2009; 146:238-244.

44. Ruyssen-Witrand A, Tubach F, Ravaud P. Systematic review reveals heterogeneity in definition of a clinically relevant difference in pain. J Clin Epidemiol 2011; 64:463-470.

45. Farrar JT, Young JP, LaMoreaux L, Werth $\mathrm{J}$, Poole RM. Clinical importance of changes in chronic pain intensity measured on an 11-point numerical pain rating scale. Pain 2001; 94:149-158.

46. Ylinen J, Takala E-P, Nykänen M, Häkkinen A, Mälkiä E, Pohjolainen T, Karppi $\mathrm{SL}$, Kautiainen $\mathrm{H}$, Airaksinen $\mathrm{O}$. Active neck muscle training in the treatment of chronic neck pain in women: A randomized controlled trial. JAMA 2003; 289:2509-2516.

47. Jay $\mathrm{K}$, Schraefel $\mathrm{M}$, Andersen $\mathrm{CH}$, Ebbesen FS, Christiansen DH, Skotte J, Zebis MK, Andersen LL. Effect of brief daily resistance training on rapid force development in painful neck and shoulder muscles: Randomized controlled trial. Clin Physiol Funct Imaging 2013; 33:386-392.

48. Jay K, Frisch D, Hansen K, Zebis MK, Andersen $\mathrm{CH}$, Mortensen OS, Andersen LL. Kettlebell training for musculoskeletal and cardiovascular health: A randomized controlled trial. Scand ] Work Env Heal 2011; 37:196-203.
49. Sundstrup E1, Jakobsen MD, Andersen $\mathrm{CH}$, Jay K, Persson R, Aagaard P, Andersen LL. Effect of two contrasting interventions on upper limb chronic pain and disability: a randomized controlled trial. Pain Physician 2014; 17:145-154.

50. Melzack R. Pain--an overview. Acta Anaesthesiol Scand 1999; 43:880-884.

51. Ye J, Cai S, Zhong W, Cai S, Zheng Q. Effects of tai chi for patients with knee osteoarthritis: A systematic review. J Phys Ther Sci 2014; 26:1133-1137.

52. Segura-Jiménez $V$, Romero-Zurita $A$, Carbonell-Baeza A, Aparicio VA, Ruiz JR, Delgado-Fernández M. Effectiveness of tai-chi for decreasing acute pain in fibromyalgia patients. Int J Sports Med 2014; 35:418-423.

53. Van Berkel J, Boot CRL, Proper KI, Bongers PM, van der Beek AJ. Effectiveness of a worksite mindfulness-based multi-component intervention on lifestyle behaviors. Int ] Behav Nutr Phys Act 2014; 11:9.

54. Carlson LE, Speca M, Patel KD, Goodey E. Mindfulness-based stress reduction in relation to quality of life, mood, symptoms of stress, and immune parameters in breast and prostate cancer outpatients. Psychosom Med 2003; 65:571-581.

55. Fjorback LO. Mindfulness and bodily distress. Dan Med ] 2012; 59:B4547.
56. Fjorback LO, Walach $\mathrm{H}$. Meditation based therapies-a systematic review and some critical observations. Religions 2012; 3:1-18.

57. Fjorback LO, Arendt M, Ornbøl E, Fink $\mathrm{P}$, Walach $\mathrm{H}$. Mindfulness-based stress reduction and mindfulness-based cognitive therapy: A systematic review of randomized controlled trials. Acta Psychiatr Scand 2011; 124:102-119.

58. Marchand WR. Mindfulness-based stress reduction, mindfulness-based cognitive therapy, and Zen meditation for depression, anxiety, pain, and psychological distress. J Psychiatr Pract 2012; 18:233-252.

59. Derbyshire SW. Exploring the pain "neuromatrix." Curr Rev Pain 2000; 4:467-477.

6o. Holzel BK, Lazar SW, Gard T, SchumanOlivier Z, Vago DR, Ott U. How does mindfulness meditation work? Proposing mechanisms of action from a conceptual and neural perspective. Perspect Psychol Sci 2011; 6:537-559.

61. Zeidan F, Martucci KT, Kraft RA, Gordon NS, McHaffie JG, Coghill RC. Brain mechanisms supporting the modulation of pain by mindfulness meditation. J Neurosci 2011; 31:5540-5548.

62. Kabat-Zinn J, Lipworth L, Burney R. The clinical use of mindfulness meditation for the self-regulation of chronic pain. J Behav Med 1985; 8:163-190. 
